\title{
Examination of the Performance of Robust Numerical Methods for Singularly Perturbed Quasilinear Problems with Interior Layers
}

\author{
P.A. Farrell and E. O'Riordan
}

\begin{abstract}
Parameter-robust numerical methods for a particular class of singularly perturbed quasilinear boundary value problems were constructed and analysed in Farrell et al. (Math Comp 78:103-127, 2009). Certain constraints were imposed in Farrell et al. (Math Comp 78:103-127, 2009) on the data to establish the final theoretical error bound. In this companion paper to Farrell et al. (Math Comp 78:103-127, 2009), the parameter-uniform performance of the numerical method is examined (via numerical experiments) when one or more of these constraints are violated. The numerical results in this paper suggest that the numerical approximations converge for a wider class of problems to that covered by the theoretical convergence analysis in Farrell et al. (Math Comp 78:103-127, 2009).
\end{abstract}

\section{Continuous Problem Class}

Convection-diffusion equations of the form $\left(-\varepsilon u_{x}\right)_{x}+(g(u))_{x}=f(x)$, with a nonlinearity of the type $g(u)=u^{2}$, arise in numerous applications involving fluid dynamics. In this paper we examine the numerical performance of parameter-robust numerical methods [1] for the following class of quasilinear singularly perturbed boundary value problems: Let $\Omega^{-}:=(0, d), \Omega^{+}:=(d, 1)$ and find $u_{\varepsilon} \in C^{1}(\bar{\Omega}) \cap$ $C^{2}\left(\Omega^{-} \cup \Omega^{+}\right)$such that

$$
\begin{gathered}
\varepsilon u_{\varepsilon}^{\prime \prime}+b(x, u) u_{\varepsilon}^{\prime}=f, \quad \text { for all } x \in \Omega^{-} \cup \Omega^{+}, \\
u_{\varepsilon}(0)=A, u_{\varepsilon}(1)=B,
\end{gathered}
$$

E. O’Riordan $(\square)$

School of Mathematical Sciences, Dublin City University, Ireland,

E-mail: eugene.oriordan@dcu.ie 


$$
\begin{gathered}
b(x, u)=\left\{\begin{array}{l}
b_{1}(u)=-1+c u, x<d \\
b_{2}(u)=1+c u, x>d
\end{array}, \quad f(x)=\left\{\begin{array}{l}
-\delta_{1}, x<d \\
\delta_{2}, x>d
\end{array}\right.\right. \\
-1<u_{\varepsilon}(0)<0,0<u_{\varepsilon}(1)<1,<c \leq 1,
\end{gathered}
$$

where $c$ is a positive constant and $\delta_{1}, \delta_{2}$ are non-negative constants. Note the strict inequalities in (1d), which are imposed in order to ensure that the solution exhibits a standard convex-concave (or S-type) shock layer, as opposed to a concave-convex (or Z-type) layer (cf. [3, pp. 15-16]).

This paper is a companion paper to [2], where asymptotic error bounds for the numerical method examined in this paper were established. In order to guarantee existence and uniqueness of the solution of the continuous problem, additional conditions on the magnitudes of $\|f\|$ and the boundary values $\left|u_{\varepsilon}(0)\right|,\left|u_{\varepsilon}(1)\right|$ were imposed in [2]. Further restrictions are required in the theoretical analysis in [2] to prove uniform in $\varepsilon$ convergence of the numerical method described below. These conditions are stated in (4) and (10).

The reduced solution $v_{0}:[0,1] \rightarrow(-1,1)$ is defined to be the solution of the following nonlinear first order problem

$$
b\left(v_{0}, x\right) v_{0}^{\prime}=f, x \in \Omega^{-} \cup \Omega^{+}, v_{0}(0)=u_{\varepsilon}(0), v_{0}(1)=u_{\varepsilon}(1) .
$$

A unique reduced solution $v_{0}$ with the additional sign-pattern property of $v_{0}(x)$ $<0, x \in \Omega^{-} ; \quad v_{0}(x)>0, x \in \Omega^{+}$exists if the conditions [2]

$$
\delta_{1} d<-u_{\varepsilon}(0)+0.5 c u_{\varepsilon}^{2}(0), \delta_{2}(1-d)<u_{\varepsilon}(1)+0.5 c u_{\varepsilon}^{2}(1)
$$

are satisfied by the data. For a unique solution of the full continuous problem to exist it suffices [2] that

$$
\begin{array}{r}
\delta_{1} d<-u_{\varepsilon}(0), \delta_{2}(1-d)<u_{\varepsilon}(1), \\
u_{\varepsilon}(1)-u_{\varepsilon}(0)<1 / c+\min \left\{\frac{\delta_{1} d}{1-c u_{\varepsilon}(0)}, \frac{\delta_{2}(1-d)}{1+c u_{\varepsilon}(1)}\right\} .
\end{array}
$$

Let $\mathbf{C}_{1}$ be the class of problems defined by (1), (3); $\mathbf{C}_{2}$ be the class of problems defined by (1), (4) and $\mathbf{C}_{3}$ be the class of problems defined by (1), (4) and (10). Note that (4a) implies (3) and hence $\mathbf{C}_{3} \subset \mathbf{C}_{2} \subset \mathbf{C}_{1}$. The proof of parameter uniform convergence of the numerical approximations given in $[2$, Theorem 6.2] restricts the problem to the smallest of these three classes $\mathbf{C}_{3}$. Figure 1 displays some typical solutions for two problems in $\mathbf{C}_{3}$, with $\varepsilon=0.000001, d=$ $0.25, \delta_{2}=0.13, u_{\varepsilon}(0)=-0.09$ and $u_{\varepsilon}(1)=0.098$. The left one is for a problem with $\delta_{1}=0.1$ and the right one for a problem with $\delta_{1}=0.35$. In this paper, we examine (via numerical experiments) the parameter-uniform performance of the numerical method when one or more of the conditions (3), (4) or (10) are violated. 

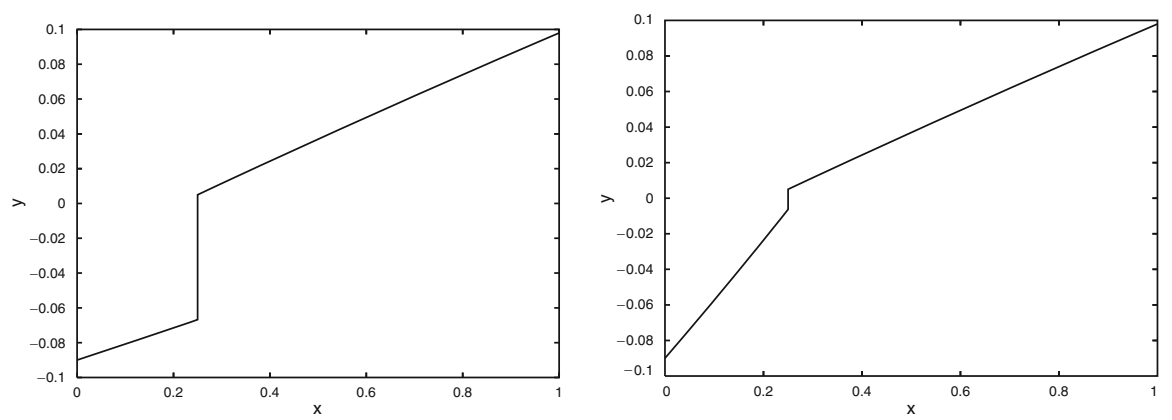

Fig. 1 Solution of (1) for sample problems in $\mathbf{C}_{3}$

Furthermore, we deduce in [2] that for the solution to a problem in $\mathbf{C}_{2}$ we have that

$$
\begin{array}{r}
\left|b_{1}\left(u_{\varepsilon}\right)\right|>\theta_{1}:=\max \left\{-c u_{\varepsilon}(0), 1-c u_{\varepsilon}(1)\right\}, x \leq d \\
b_{2}\left(u_{\varepsilon}\right)>\theta_{2}:=\max \left\{c u_{\varepsilon}(1), 1+c u_{\varepsilon}(0)\right\}, x \geq d .
\end{array}
$$

Lemma 1 ([2]). Assume the problem is in $\mathbf{C}_{2}$. The solution can be written as a linear sum of the form $u_{\varepsilon}=v_{\varepsilon}+w_{\varepsilon}$, where for each integer $k$, satisfying $1 \leq k \leq 3$, these components satisfy the following bounds,

$$
\begin{gathered}
\left\|v_{\varepsilon}\right\| \leq C,\left\|v_{\varepsilon}^{(k)}\right\|_{\Omega^{-} \cup \Omega^{+}} \leq C\left(1+\varepsilon^{2-k}\right), \\
\left|\left[v_{\varepsilon}\right](d)\right| \leq C,\left|\left[v_{\varepsilon}^{\prime}\right](d)\right| \leq C,\left|\left[v_{\varepsilon}^{\prime \prime}\right](d)\right| \leq C, \\
\left|w_{\varepsilon}^{(k)}(x)\right| \leq \begin{cases}C \varepsilon^{-k} e^{-(d-x) \theta_{1} / \varepsilon}, & x \in \Omega^{-}, \\
C \varepsilon^{-k} e^{-(x-d) \theta_{2} / \varepsilon}, & x \in \Omega^{+},\end{cases}
\end{gathered}
$$

where $C$ is a constant independent of $\varepsilon$.

\section{Numerical Method}

The domain $\bar{\Omega}$ is subdivided into the four subintervals

$$
\left[0, d-\sigma_{1}\right] \cup\left[d-\sigma_{1}, d\right] \cup\left[d, d+\sigma_{2}\right] \cup\left[d+\sigma_{2}, 1\right],
$$

for some $\sigma_{1}, \sigma_{2}$ that satisfy $0<\sigma_{1} \leq \frac{d}{2}, 0<\sigma_{2} \leq \frac{1-d}{2}$. On each of the four subintervals a uniform mesh with $\frac{N}{4}$ mesh-intervals is placed. The interior mesh points are denoted by

$$
\Omega_{\varepsilon}^{N}:=\left\{x_{i}: 1 \leq i \leq N-1, i \neq N / 2\right\} .
$$


Clearly $x_{\frac{N}{2}}=d, \bar{\Omega}_{\varepsilon}^{N}=\left\{x_{i}\right\}_{0}^{N}$ and $\sigma_{1}, \sigma_{2}$ are taken to be the following

$$
\sigma_{1}:=\min \left\{\frac{d}{2}, 2 \frac{\varepsilon}{\theta_{1}} \ln N\right\}, \sigma_{2}:=\min \left\{\frac{1-d}{2}, 2 \frac{\varepsilon}{\theta_{2}} \ln N\right\},
$$

whose choice can be motivated from (5) and the earlier bounds on $w_{\varepsilon}^{(k)}$. Then the fitted mesh method for problem (1) is: Find a mesh function $U_{\varepsilon}$ such that

$$
\begin{array}{r}
\varepsilon \delta^{2} U_{\varepsilon}\left(x_{i}\right)+b\left(x_{i}, U_{\varepsilon}\left(x_{i}\right)\right) D U_{\varepsilon}\left(x_{i}\right)=f\left(x_{i}\right) \quad \text { for all } \quad x_{i} \in \Omega_{\varepsilon}^{N} \\
U_{\varepsilon}(0)=u_{\varepsilon}(0), \quad U_{\varepsilon}(1)=u_{\varepsilon}(1) \\
D^{-} U_{\varepsilon}\left(x_{\frac{N}{2}}\right)=D^{+} U_{\varepsilon}\left(x_{\frac{N}{2}}\right)
\end{array}
$$

where

$$
\delta^{2} Z_{i}=\frac{D^{+} Z_{i}-D^{-} Z_{i}}{\left(x_{i+1}-x_{i-1}\right) / 2}, \quad D Z_{i}= \begin{cases}D^{-} Z_{i}, & i<N / 2, \\ D^{+} Z_{i}, & i>N / 2,\end{cases}
$$

$D^{+}$and $D^{-}$are the standard forward and backward finite difference operators, respectively. In order to solve this nonlinear finite difference scheme we use a variant of the continuation method from [1, Sect. 10.3].

$$
\begin{gathered}
\left(\varepsilon \delta_{x}^{2}+b\left(x_{i}, U_{\varepsilon}\left(x_{i}, t_{j-1}\right)\right) D-D_{t}^{-}\right) U_{\varepsilon}\left(x_{i}, t_{j}\right)=f\left(x_{i}\right), x_{i} \neq d, j=1, \ldots K \\
D_{x}^{-} U_{\varepsilon}\left(d, t_{j}\right)=D_{x}^{+} U_{\varepsilon}\left(d, t_{j}\right), j=1, \ldots K \\
U_{\varepsilon}\left(0, t_{j}\right)=u_{\varepsilon}(0), U_{\varepsilon}\left(1, t_{j}\right)=u_{\varepsilon}(1) \text { for all } j \\
U_{\varepsilon}(x, 0)=u(0)+(u(1)-u(0)) x
\end{gathered}
$$

and $D_{t}^{-}$is the standard backward finite difference operator in time. The choices of the uniform time-like step $k=t_{j}-t_{j-1}$ and the number of iterations $K$ are determined as follows. Defining

$$
e(j):=\max _{1 \leq i \leq N}\left|U_{\varepsilon}\left(x_{i}, t_{j}\right)-U_{\varepsilon}\left(x_{i}, t_{j-1}\right)\right| / k, \quad \text { for } j=1,2, \cdots, K
$$

the time-like step $k$ is chosen sufficiently small so that

$$
e(j) \leq e(j-1), \quad \text { for all } j \text { satisfying } 1<j \leq K .
$$

Then the number of iterations $K$ is chosen such that

$$
e(K) \leq \mathrm{TOL}:=10^{-7}
$$

The numerical solution is computed using the following algorithm. Start from $t_{0}$ with the initial timestep $k=1.0$. If, at some value of $j,(9 \mathrm{~b})$ is not satisfied, then discard the timestep from $t_{j-1}$ to $t_{j}$ and restart from $t_{j-1}$ with half the time step, that is $k^{n e w}=k / 2$, and continue halving the timestep until one finds a $k$ for which $(9 \mathrm{~b})$ is satisfied. Assuming that (9b) is satisfied at each timestep, continue until either (9c) is satisfied or $t_{j}=1,000$. If (9c) is not satisfied, we repeat the entire process 
again from $t_{0}$, halving the initial timestep $k$ to $k=0.5$. If the process still stalls, we restart from $t_{0}$ again halving the initial timestep. If $(9 \mathrm{c})$ is satisfied the resulting values of $U_{\varepsilon}(x, K)$ are taken as the approximations to the solution of the continuous problem.

The same conditions required for existence of the solution of the full continuous problem are also sufficient for the existence (but not uniqueness) of the solution of the discrete nonlinear problem.

In [2], it is established that, providing $N$ is sufficiently large and $\varepsilon$ is sufficiently small, independently of each other, under the further implicit restriction that

$$
b^{2}\left(x_{i}, U_{\varepsilon}\right)-4 \varepsilon c u_{\varepsilon}^{\prime}>0, \quad x_{i} \neq d,
$$

we can prove a uniform in $\varepsilon$ error bound at all the mesh points of the form

$$
\left\|U_{\varepsilon}-u_{\varepsilon}\right\|_{\Omega} \leq C N^{-1}(\ln N)^{2},
$$

where $u_{\varepsilon}$ is the continuous solution, $U_{\varepsilon}$ is a discrete solution of (7), and $C$ is a constant independent of $N$ and $\varepsilon$. The condition (10) is implicit as the exact solution $u_{\varepsilon}$ is, in general, unknown.

\section{Robustness of the Solution Method}

Example 1. For the uniform convergence result (11) to be valid, [2] requires that (4) and (10) must be satisfied. For example, if

$$
c=1, \quad \delta_{1} d<-u_{\varepsilon}(0)<0.1 \quad \text { and } \quad \delta_{2}(1-d)<u_{\varepsilon}(1)<0.1
$$

then the data constraints (4) and (10) in $\mathbf{C}_{3}$ are both satisfied. Thus a problem with

$$
d=0.25, \delta_{2}=0.13, \delta_{1}<0.4,0.0975<u_{\varepsilon}(1)<0.1,-0.1<u_{\varepsilon}(0)<-\delta_{1} / 4
$$

satisfies these constraints. We consider a problem with $\mathrm{u}(0)=-0.09, \mathrm{u}(1)=0.098$, $\delta_{2}=0.13$ and $\delta_{1}$ varying from 0.1 to 0.35 . This choice for the data satisfies all three assumptions including the implicit one (10). We verify this assertion numerically by computing

$$
T_{\varepsilon}^{N}\left(x_{i}\right)= \begin{cases}b^{2}\left(x_{i}, U_{\varepsilon}^{N}\right)-4 \varepsilon D^{-} U_{\varepsilon}^{N}, & x_{i}<d \\ b^{2}\left(x_{i}, U_{\varepsilon}^{N}\right)-4 \varepsilon D^{+} U_{\varepsilon}^{N}, & x_{i}>d\end{cases}
$$

and observing that $T_{\varepsilon}^{N}=\min _{i} T_{\varepsilon}^{N}\left(x_{i}\right)>0$ for all values of $\varepsilon$ and $N$ used. The computed uniform rates of convergence $p_{N}$, using the double mesh principle and the uniform fine mesh errors $E_{N}$ (see [1, pp. 104, 190] for details on how these quantities are calculated) are computed over the range $\varepsilon=2^{-j}, j=1,2, \ldots 25$ and are presented in Table 1 . These results confirm uniform convergence in this range of the data. 
Table 1 Maximum errors $E_{N}$ and computed rates of convergence $p_{N}$ for the numerical method (6), (7) for problems within $\mathbf{C}_{\mathbf{3}}$ in the case of Example 1

\begin{tabular}{|c|c|c|c|c|c|c|}
\hline$N$ & 32 & 64 & 128 & 256 & 512 & 1,024 \\
\hline \multicolumn{7}{|c|}{$\delta_{1}=0.1$} \\
\hline$E_{N}$ & 0.004962 & 0.003227 & 0.002017 & 0.001175 & 0.000637 & 0.000313 \\
\hline$p_{N}$ & 0.46 & 0.75 & 0.63 & 0.72 & 0.68 & 0.84 \\
\hline \multicolumn{7}{|c|}{$\delta_{1}=0.2$} \\
\hline$E_{N}$ & 0.003583 & 0.002245 & 0.001346 & 0.000771 & 0.000413 & 0.000201 \\
\hline$p_{N}$ & 0.57 & 0.76 & 0.72 & 0.72 & 0.72 & 0.85 \\
\hline \multicolumn{7}{|c|}{$\delta_{1}=0.3$} \\
\hline$E_{N}$ & 0.002549 & 0.001403 & 0.000809 & 0.000457 & 0.000243 & 0.000117 \\
\hline$p_{N}$ & 0.70 & 0.90 & 0.79 & 0.76 & 0.73 & 0.86 \\
\hline \multicolumn{7}{|c|}{$\delta_{1}=0.35$} \\
\hline$E_{N}$ & 0.002205 & 0.001151 & 0.000584 & 0.000295 & 0.000155 & 0.000075 \\
\hline$p_{N}$ & 0.90 & 0.94 & 0.96 & 0.93 & 0.72 & 0.88 \\
\hline
\end{tabular}

Table 2 Maximum errors $E_{N}$ and computed rates of convergence $p_{N}$ for a problem outside $\mathbf{C}_{1}$, but satisfying (10), in the case of Example 1

\begin{tabular}{lllllll}
\hline \multicolumn{7}{c}{$\delta_{1}=0.39$} \\
\hline$N$ & 32 & 64 & 128 & 256 & 512 & 1,024 \\
\hline$E_{N}$ & 0.002282 & 0.001154 & 0.000578 & 0.000283 & 0.000133 & 0.000057 \\
$p_{N}$ & 0.98 & 0.96 & 0.98 & 0.99 & 0.99 & 1.00 \\
\hline
\end{tabular}

Now consider the same problem with $u(0)=-0.09, u(1)=0.098, \delta_{2}=0.13$ and $\delta_{1}=0.39$. This does not satisfy (3) and hence is not in $\mathbf{C}_{1}$. However, this scheme does numerically satisfy the implicit condition (10).

The results presented in Table 2 imply that the scheme is still convergent uniformly in $\varepsilon$.

Example 2. For the existence of a continuous solution we have the sufficient conditions (4). As an example, take

$$
c=1, u_{\varepsilon}(1)=0.7, u_{\varepsilon}(0)=-0.5 d=0.25 \text {. }
$$

Then (3) is satisfied when $\delta_{1}<2.5$ and $\delta_{2}<1.26$. Also (4a) is satisfied when

$$
\delta_{1}<2 \text { and } \delta_{2}<\frac{2.8}{3} \approx 0.933333
$$

and (4b) is satisfied when

$$
\delta_{1}>1.2 \text { and } \delta_{2}>\frac{1.36}{3} \approx 0.453333 \text {. }
$$



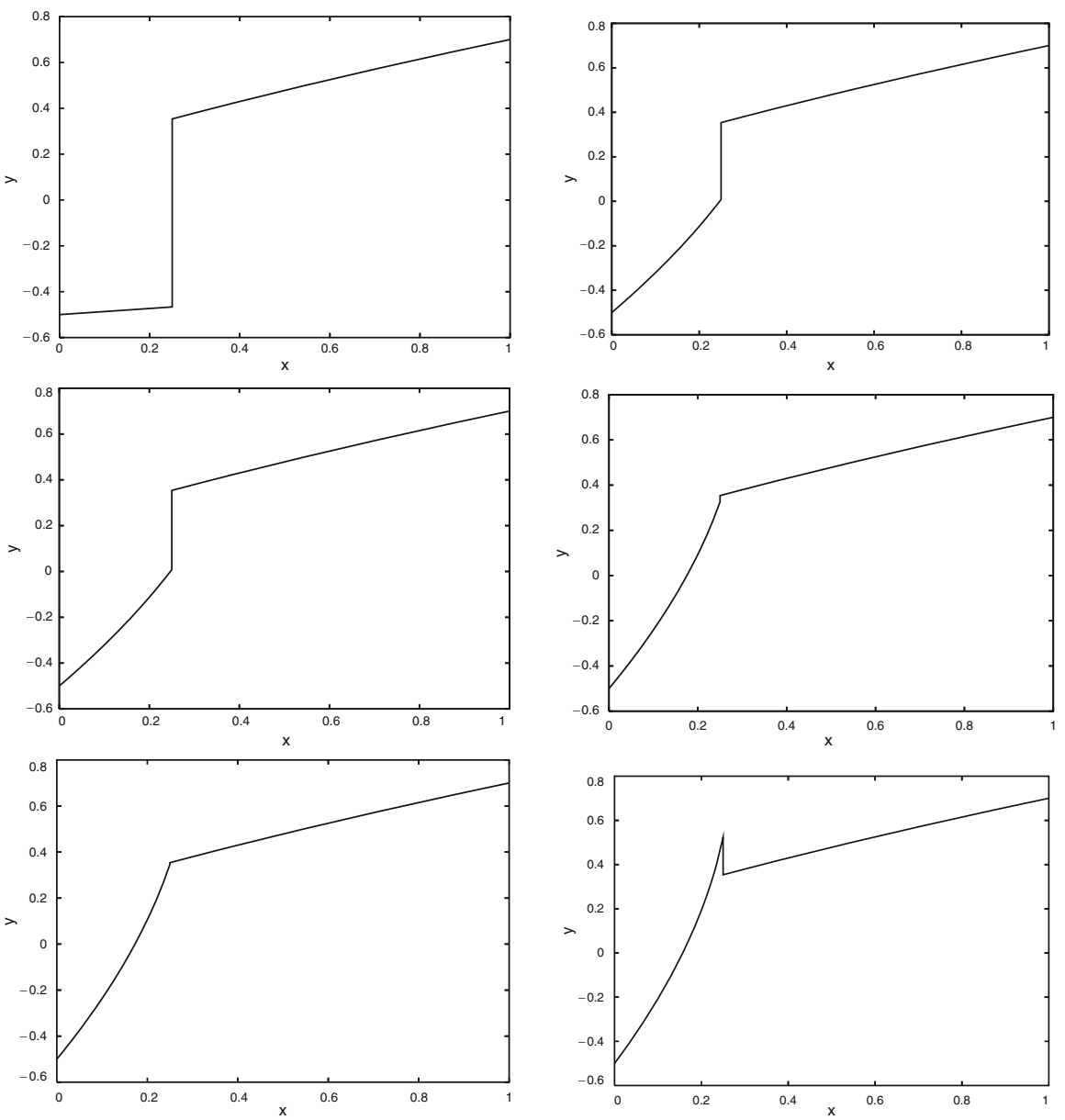

Fig. 2 Solution of (1) for problems which do not satisfy $\mathbf{C}_{3}$. In all these figures, $\delta_{2}=$ $0.7, u(0)=-0.5, u(1)=0.7, N=64$ and $\varepsilon=0.000001$. From top left to bottom right: $\delta_{1}$ $=0.2,2.4999,2.5,3.5,3.55,3.9$

We fix $\delta_{2}=0.7$ and consider various values of $\delta_{1}$, in particular ones which violate one or more of the conditions (3), (4a) or (4b). For the problems examined in this example, it has been observed numerically, using condition (12), that the implicit condition (10) is not satisfied for any of the values of $\delta_{1}$ considered. That is, these problems lie outside the class $\mathbf{C}_{3}$. Problems are in the class $\mathbf{C}_{2} \backslash \mathbf{C}_{3}$ if $1.2<\delta_{1}<2$, in the class $\mathbf{C}_{1} \backslash \mathbf{C}_{2}$ if $2 \leq \delta_{1}<2.5$ or if $\delta_{1} \leq 1.2$ and finally the problem lies outside $\mathrm{C}_{1}$ if $\delta_{1} \geq 2.5$.

Illustrations of the corresponding solutions are given in Fig. 2, and the convergence results are given in Tables 3-5. They show that provided the reduced solution 
Table 3 Maximum errors $E_{N}$ and computed rates of convergence $p_{N}$ for the numerical method (6), (7) applied to problems in $\mathbf{C}_{2}$, where (10) is violated, that is within $\mathbf{C}_{2} \backslash \mathbf{C}_{\mathbf{3}}$ in the case of Example 2 with $\delta_{2}=0.7$

\begin{tabular}{lllllll}
\hline$N$ & 32 & 64 & 128 & 256 & 512 & 1,024 \\
\hline \multicolumn{5}{c}{$E_{N}=1.3$} \\
$\boldsymbol{E}_{N}$ & 0.067928 & 0.053165 & 0.033076 & 0.020709 & 0.011692 & 0.005732 \\
& 0.09 & 0.65 & 0.71 & 0.57 & 0.74 & 0.71 \\
$E_{N}$ & 0.058642 & 0.047114 & 0.029970 & 0.018685 & 0.010404 & 0.005133 \\
$p_{N}$ & 0.13 & 0.66 & 0.73 & 0.56 & 0.70 & 0.71 \\
\hline
\end{tabular}

Table 4 Maximum errors $E_{N}$ and computed rates of convergence $p_{N}$ for the numerical method (6), (7) applied to problems in $\mathbf{C}_{\mathbf{1}}$, where (4) and (10) are violated, that is within $\mathbf{C}_{\mathbf{1}} \backslash \mathbf{C}_{\mathbf{2}}$ in the case of Example 2 with $\delta_{2}=0.7$

\begin{tabular}{|c|c|c|c|c|c|c|}
\hline$N$ & 32 & 64 & 128 & 256 & 512 & 1,024 \\
\hline \multicolumn{7}{|c|}{$\delta_{1}=0.2$} \\
\hline$E_{N}$ & 0.085977 & 0.070653 & 0.045129 & 0.028786 & 0.016281 & 0.008038 \\
\hline$p_{N}$ & 0.01 & 0.62 & 0.70 & 0.55 & 0.70 & 0.70 \\
\hline \multicolumn{7}{|c|}{$\delta_{1}=0.5$} \\
\hline$E_{N}$ & 0.081286 & 0.063318 & 0.039899 & 0.025084 & 0.014299 & 0.007035 \\
\hline$p_{N}$ & 0.00 & 0.62 & 0.70 & 0.56 & 0.74 & 0.70 \\
\hline \multicolumn{7}{|c|}{$\delta_{1}=1.1$} \\
\hline$E_{N}$ & 0.071339 & 0.055289 & 0.034691 & 0.021476 & 0.012067 & 0.005918 \\
\hline$p_{N}$ & 0.08 & 0.65 & 0.71 & 0.57 & 0.76 & 0.71 \\
\hline \multicolumn{7}{|c|}{$\delta_{1}=2.1$} \\
\hline$E_{N}$ & 0.052495 & 0.042713 & 0.027518 & 0.016995 & 0.009474 & 0.004675 \\
\hline$p_{N}$ & 0.16 & 0.68 & 0.73 & 0.57 & 0.69 & 0.71 \\
\hline \multicolumn{7}{|c|}{$\delta_{1}=2.4$} \\
\hline$E_{N}$ & 0.045858 & 0.037679 & 0.024406 & 0.014925 & 0.008380 & 0.004132 \\
\hline$p_{N}$ & 0.21 & 0.68 & 0.74 & 0.59 & 0.67 & 0.72 \\
\hline \multicolumn{7}{|c|}{$\delta_{1}=2.4999$} \\
\hline$E_{N}$ & 0.043529 & 0.035851 & 0.023213 & 0.014147 & 0.007960 & 0.003927 \\
\hline$p_{N}$ & 0.23 & 0.67 & 0.74 & 0.60 & 0.68 & 0.72 \\
\hline
\end{tabular}

of the problem remains monotonic increasing, the method is robust in the sense that the numerical method remains uniformly in $\varepsilon$ convergent. When the problem ceases to be monotonic the layer type changes from a standard shock layer to a Z-layer. As the Z-layer grows in amplitude the nonlinear solver does not converge and thus the method ceases to be robust. 
Table 5 Maximum errors $E_{N}$ and computed rates of convergence $p_{N}$ for the numerical method (6), (7) applied to problems outside $\mathbf{C}_{\mathbf{1}}$, that is where (3), (4) and (10) are violated, in the case of Example 2 with $\delta_{2}=0.7$

\begin{tabular}{|c|c|c|c|c|c|c|}
\hline$N$ & 32 & 64 & 128 & 256 & 512 & 1,024 \\
\hline \multicolumn{7}{|c|}{$\delta_{1}=2.8$} \\
\hline$E_{N}$ & 0.041487 & 0.029870 & 0.019123 & 0.011529 & 0.006529 & 0.003246 \\
\hline$p_{N}$ & 0.39 & 0.64 & 0.77 & 0.65 & 0.68 & 0.71 \\
\hline \multicolumn{7}{|c|}{$\delta_{1}=3.0$} \\
\hline$E_{N}$ & 0.043328 & 0.025441 & 0.015947 & 0.009703 & 0.005490 & 0.002714 \\
\hline$p_{N}$ & 0.83 & 0.63 & 0.79 & 0.69 & 0.68 & 0.71 \\
\hline \multicolumn{7}{|c|}{$\delta_{1}=3.5$} \\
\hline$E_{N}$ & 0.075558 & 0.032340 & 0.015213 & 0.007286 & 0.003408 & 0.001470 \\
\hline$p_{N}$ & 1.32 & 1.12 & 1.04 & 1.00 & 0.99 & 0.98 \\
\hline \multicolumn{7}{|c|}{$\delta_{1}=3.8$} \\
\hline$E_{N}$ & 0.168256 & 0.056174 & 0.024782 & 0.011446 & 0.005227 & 0.002217 \\
\hline$p_{N}$ & 1.84 & 1.24 & 1.10 & 1.05 & 1.02 & 1.01 \\
\hline
\end{tabular}

\section{Sensitivity to the Position of the Transition Points}

We examine the effect of varying the fine mesh width by incorporating a constant $C_{*}$ in a revised formula for $\sigma_{1}$ and $\sigma_{2}$ given by

$$
\sigma_{1}=\min \left\{\frac{d}{2}, C_{*} \frac{\varepsilon}{\theta_{1}} \ln N\right\}, \sigma_{2}=\min \left\{\frac{1-d}{2}, C_{*} \frac{\varepsilon}{\theta_{2}} \ln N\right\},
$$

where $C_{*}$ is a parameter and $\theta_{1}, \theta_{2}$ are specified in (5).

Table 6 give the results for Example 2 with $\delta_{1}=1.20010$. For the range of $C_{*}$ tested, it was observed that the number of iterations varied by at most a factor of two.

Thus the method is not particularly sensitive to the fine mesh width and, in fact, a choice of a value of $C_{*}$ less than that of $C_{*}=2$ used in [2] seems to give better performance. In the example considered here, the errors are smallest and the rate of convergence best for $C_{*}=0.5$.

Remark 1. The theoretical rate of convergence given in (11) can be compared to the observed rates of convergence given in Tables 1-6, by using Table 7. For example, Table 1 exhibits rates close to $N^{-1} \ln N$ and Tables 3-6 mainly exhibit rates close to $N^{-1}(\ln N)^{2}$. 
Table 6 Maximum errors $E_{N}$ and computed rates of convergence $p_{N}$ for various choices of the transition point in the case of Example 2 with $\delta_{1}=1.20010, \delta_{2}=0.7$

\begin{tabular}{|c|c|c|c|c|c|c|}
\hline$N$ & 32 & 64 & 128 & 256 & 512 & 1,024 \\
\hline \multicolumn{7}{|c|}{$C_{*}=0.125$} \\
\hline$E_{N}$ & 0.077109 & 0.063909 & 0.052342 & 0.040499 & 0.028576 & 0.017859 \\
\hline$p_{N}$ & 0.37 & 0.34 & 0.27 & 0.24 & 0.26 & 0.27 \\
\hline \multicolumn{7}{|c|}{$C_{*}=0.25$} \\
\hline$E_{N}$ & 0.055713 & 0.034658 & 0.020660 & 0.011906 & 0.006556 & 0.003274 \\
\hline$p_{N}$ & 0.70 & 0.68 & 0.71 & 0.71 & 0.71 & 0.70 \\
\hline \multicolumn{7}{|c|}{$C_{*}=0.5$} \\
\hline$E_{N}$ & 0.039241 & 0.021406 & 0.012181 & 0.006681 & 0.003483 & 0.001645 \\
\hline$p_{N}$ & 0.81 & 0.89 & 0.79 & 0.80 & 0.82 & 0.78 \\
\hline \multicolumn{7}{|c|}{$C_{*}=1.0$} \\
\hline$E_{N}$ & 0.052324 & 0.033291 & 0.020706 & 0.011990 & 0.006454 & 0.003099 \\
\hline$p_{N}$ & 0.23 & 0.79 & 0.68 & 0.73 & 0.77 & 0.76 \\
\hline \multicolumn{7}{|c|}{$C_{*}=2.0$} \\
\hline$N$ & 32 & 64 & 128 & 256 & 512 & 1,024 \\
\hline$E_{N}$ & 0.069652 & 0.054194 & 0.033899 & 0.021033 & 0.011889 & 0.005824 \\
\hline$p_{N}$ & 0.08 & 0.65 & 0.71 & 0.57 & 0.75 & 0.71 \\
\hline
\end{tabular}

Table 7 Orders of local convergence $p^{N}$ corresponding to different theoretical error bounds for various values of $N$

\begin{tabular}{lllllll}
\hline$N$ & 32 & 64 & 128 & 256 & 512 & 1,024 \\
\hline$N^{-1} \ln N$ & 0.68 & 0.74 & 0.78 & 0.81 & 0.83 & 0.85 \\
$N^{-1}(\ln N)^{2}$ & 0.28 & 0.44 & 0.53 & 0.60 & 0.65 & 0.69 \\
\hline
\end{tabular}

\section{Conclusions}

The numerical results in this paper indicate a possible gap between the theory in [2] and what is observed in practice. As was proven in [2] the scheme (6), (7) is a parameter-uniform scheme under the conditions (4) and (10). However these sufficient conditions appear to be overly restrictive, since, in practice, the numerical approximations appear to converge for a wider range of data. In any attempt to extend the theory in [2] to a wider class of problems, a reasonable constraint on the data to aim for (in place of (4)) would be that the reduced solution is monotonic increasing, which is a necessary condition to exclude Z-layers from appearing in the solution of (1).

The implicit condition (10) is not satisfied for some of the examples presented here, while the numerical approximations still converge uniformly in $\varepsilon$. When the constraint (10) is violated it appears that $T_{\varepsilon}^{N}\left(x_{i}\right)<0$ in a particular neighborhood of the point $d$ and not at the transition points between the fine and coarse mesh. Proving convergence without (10) being satisfied would require a method of 
proof other than the maximum principle arguments used in [2]. These numerical results also suggest that a different finite difference equation (other than continuity of the discrete first derivative) at the point of the discontinuity $d$ may ensure that $T_{\varepsilon}^{N}>0$, which in turn might improve the performance of the scheme and also assist in extending the scope of the current theory.

\section{References}

1. P.A. Farrell, A.F. Hegarty, J.J.H. Miller, E. O'Riordan, and G.I. Shishkin, Robust Computational Techniques for Boundary Layers, Chapman and Hall/CRC, New York/Boca Raton, (2000)

2. P.A. Farrell, E. O'Riordan, and G.I. Shishkin, A class of singularly perturbed quasilinear differential equations with interior layers, Mathematics of Computation 78(265):103-127 (2009)

3. F.A. Howes, Boundary-interior layer interactions in nonlinear singular perturbation theory, Memoirs of the AMS 15:203 (1978) 